\title{
Advances in the Structural Evaluation of Glaucoma with Optical Coherence Tomography
}

\author{
Daniel Meira-Freitas • Renato Lisboa • \\ Felipe A. Medeiros
}

Published online: 22 March 2013

(c) Springer Science + Business Media New York 2013

\begin{abstract}
Optical coherence tomography (OCT) is capable of providing quantitative and objective assessments of the optic disc, macula and retinal nerve fiber layer in glaucoma. The recent advent of spectral domain OCT (SD-OCT) has enhanced the resolution, decreased scan acquisition time, and improved the reproducibility of measurements compared to older versions of this technology. However, although OCT has been successfully used for detection of disease and evaluation of progression, the limited agreement between structural and functional tests indicates the strong need for a combined approach for detecting and monitoring the disease. A recently described approach for estimation of rates of retinal ganglion cell loss from a combination of SDOCT and functional data is a promising method for diagnosing, staging, detecting progression, and estimating rates of glaucomatous deterioration.
\end{abstract}

Keywords Glaucoma - Optic nerve - Optic disk · Optical coherence tomography $\cdot$ Retina .

Retinal ganglion cells

\section{Introduction}

Glaucoma is the leading cause of irreversible blindness and visual impairment in the world. The disease is characterized

D. Meira-Freitas · R. Lisboa · F. A. Medeiros $(\bowtie)$

Hamilton Glaucoma Center, Department of Ophthalmology,

University of California, San Diego, 9500 Gilman Drive,

La Jolla, CA 92093, USA

e-mail: fmedeiros@glaucoma.ucsd.edu

D. Meira-Freitas · R. Lisboa

Department of Ophthalmology, Federal University of São Paulo, São Paulo, Brazil by progressive retinal ganglion cell (RGC) loss with associated structural changes at the level of the optic nerve and retinal nerve fiber layer (RNFL). In the past two decades, a large volume of evidence has accumulated with regard to the role of structural measurements of the topography of the optic nerve head $(\mathrm{ONH})$ and RNFL thickness for diagnosing and detecting glaucoma progression. There is now substantial evidence that many patients develop structural changes before the appearance of detectable loss in standard functional tests $[1 \cdot, 2-7]$. Therefore, technologies that can objectively evaluate the optic nerve using a noninvasive approach are essential for the early diagnosis and follow-up of the disease.

Optical coherence tomography (OCT) is one of the most studied technologies among the methods that provide an objective and reliable structural evaluation of the $\mathrm{ONH}$. The introduction of spectral domain OCT (SD-OCT) has greatly enhanced the resolution, decreased scan acquisition time $[8,9]$, and improved reproducibility compared to the previous time domain OCT (TD-OCT) [10,11], potentially improving the ability to detect and monitor the disease. Moreover, recent advances in retinal segmentation algorithms with SD-OCT have made possible the automated detection of boundaries of individual retinal layers in the macular region, providing a new tool for glaucoma assessment [12-17].

This review discusses the recent advances in structural evaluation of the optic nerve and retina by SD-OCT for glaucoma. It also discusses recent developments, such as the new combined structure and function index (CSFI) for diagnosing and assessing progressive glaucomatous damage.

\section{Working Principles of the SD-OCT Technology}

Three of the current commercially available SD-OCT devices are the RTVue (Optovue, Fremont, California, 
USA), the Cirrus (Carl Zeiss Meditec, Dublin, CA, USA) and the Spectralis (Heidelberg Engineering, Dossenheim, Germany). The principles of imaging acquisition are similar for each of these devices and involve the use of a scanning laser diode to provide high-resolution images of neuroretinal tissue through the detection of the echo optical signal measured by the spectral/Fourier domain technique [18]. Previous studies have demonstrated that measurements taken from these images show good clinical-histological correlations [19-21].

The RTVue uses a scanning laser diode of $840 \mathrm{~nm}$ wavelength to provide images with an acquisition rate of 26,000 A-scans per second and has a 5- $\mu \mathrm{m}$ depth resolution. The ONH scan protocol generates a polar RNFL thickness map that is measured along a circle of $3.45 \mathrm{~mm}$ in diameter centered on the optic disc [22]. The ONH protocol also performs 12 radial scans (452 A-scans each) and 6 concentric ring scans ranging from 2.5 to $4.0 \mathrm{~mm}$ in diameter (587-775 A-scans each) centered on the optic disc to obtain topographic measurements. Areas between A-scans are interpolated [23]. The ganglion cell complex (GCC) scan protocol generates a thickness map of the three innermost retinal layers of the macula: the nerve fiber, ganglion cell, and inner plexiform layers. The GCC protocol samples the macula over a $7-\mathrm{mm}^{2}$ area with its scan center shifted $0.75 \mathrm{~mm}$ temporally to improve sampling of the temporal periphery [16].

The Cirrus OCT uses a superluminescent diode laser of $840 \mathrm{~nm}$ wavelength with an acquisition rate of 27,000 A-scans per second and has a $5-\mu \mathrm{m}$ depth resolution. The optic disc cube protocol provides RNFL thickness measurements and is based on a tridimensional scan of a $6 \times 6-\mathrm{mm}^{2}$ area centered on the optic disc, where information from 1,024 (depth) $\times 200 \times 200$-point parallelepiped is collected. Then, the information about the RNFL thickness is obtained from the 3.46-mm-diameter circumference around the optic disc [22]. For the detection of the inner macular layers, the macular cube $200 \times 200$ scan protocol is processed with the ganglion cell analysis (GCA) algorithm. This protocol performs 200 horizontal B-scans comprising $200 \mathrm{~A}$-scans per B-scan over 1,024 samplings within a cube measuring $6 \times 6 \times 2 \mathrm{~mm}$. The GCA algorithm identifies the outer boundary of the RNFL and the outer boundary of the inner plexiform layer [17].

Spectralis OCT uses a dual-beam SD-OCT and a confocal scanning laser ophthalmoscope (CSLO) that employs a scanning laser diode of $870 \mathrm{~nm}$ wavelength with an acquisition rate of 40,000 A-scans per second and has a 3.9- $\mu \mathrm{m}$ depth resolution. The RNFL circle scan consists of 1,024 A-scan points from a $3.45-\mathrm{mm}$ circle centered on the optic disc [22]. The Spectralis OCT currently does not have OCT protocols to evaluate topographic parameters of the optic disc and to detect the macular GCC.
Although RNFL thickness measurements obtained by the different SD-OCT instruments are highly correlated, they are not interchangeable [10, 22, 24, 25]. For example, RTVue measurements are consistently thicker than Cirrus and Spectralis for average and quadrant-wise RNFL thickness [22]. This may be attributed in part to differences in RNFL detection algorithms. Therefore, it is important to keep in mind these differences when patients had measurements obtained by different instruments during follow-up.

\section{SD-OCT for Glaucoma Diagnosis}

Even though SD-OCT instruments have different hardware and acquisition protocols, measurements of RNFL with Spectralis, Cirrus, and RTVue show similar accuracies for discrimination between perimetric glaucoma and healthy eyes [26]. In most studies, the RNFL parameters with best diagnostic performance are the inferior quadrant thickness, average thickness, and superior quadrant thickness [23, 27-31]. This is an expected finding, as the pattern of glaucomatous damage typically affects the superior and inferior regions of the optic disc. Among the ONH topographic parameters, those with the best diagnostic performance are rim area, vertical cup-disc ratio [29, 30], and vertical rim thickness [30]. As expected, GCC thickness parameters have been shown to perform better than total macular retinal thickness in differentiating between perimetric glaucoma and healthy eyes $[16,32]$.

RNFL, ONH topographic, and macular thickness protocols have been compared. Reported results diverge between different SD-OCT devices. Studies with the RTVue for differentiating perimetric glaucoma from normal eyes showed that RNFL and GCC parameters had similar diagnostic accuracy, whereas both performed better than $\mathrm{ONH}$ parameters [23, 32]. This could reflect a superior performance of RNFL and macular assessment for detection of glaucomatous damage. In fact, the $\mathrm{ONH}$ structure is likely to have greater variability than the RNFL and macular areas due to the presence of tilting, sloping, crescents, and areas of parapapillary atrophy, and this can potentially influence the diagnostic ability of topographic measurements. Alternatively, the inferior performance of topographic parameters could reflect a relatively weaker algorithm for assessing ONH topography built in the RTVue system.

On the other hand, a study that compared diagnostic accuracies of ONH and RNFL parameters using Cirrus OCT for differentiating perimetric glaucoma from normal eyes did not find significant differences between these two scanning areas [30]. Comparison between RNFL and GCC assessment with Cirrus OCT indicated that RNFL measurements were generally superior when used to diagnose glaucoma; nevertheless, macular thickness parameters were of greater value in 
specific situations, such as in eyes with larger optic discs [33]. As described previously, Cirrus and RTVue OCTs use different methods to capture the image of the $\mathrm{ONH}$, and this may explain the differences in the studies. Differences could also be explained by different inclusion/exclusion criteria for the studies and severity of disease in the glaucomatous population. Further studies are necessary evaluating the performance of these scanning areas in the same population.

The SD-OCT diagnostic ability has been compared to other imaging technologies. The RNFL assessment with Spectralis OCT showed better performance than topographic disc assessment with confocal scanning laser ophthalmoscopy [Heidelberg Retinal Tomograph (HRT); Heidelberg Engineering], to detect perimetric [28] and pre-perimetric [34] glaucoma. Although the RNFL measurements with RTVue and GDx VCC (Carl Zeiss Meditec) had a good correlation, the RNFL thickness obtained by RTVue OCT showed statistically significant better performance in distinguishing perimetric glaucoma from normal subjects in one investigation [35].

\section{Structural Evaluation for Detection of Progression of Glaucomatous Damage}

High resolution and low variability are essential requirements for the detection of glaucomatous progression with imaging devices. SD-OCT devices show excellent reproducibility for RNFL measurements in healthy and glaucomatous eyes [24, 36]. Studies comparing the reproducibility of SD-OCT and TD-OCT indicated that the measurement variability of RNFL thickness was significantly lower with SD-OCT [10, 11, 37]. Studies of macular segmentation reproducibility with Cirrus OCT [15] and RTVue OCT [16] have also reported low variability.

Although most glaucoma patients will show some evidence of progression if followed for long enough, the rate of deterioration can be highly variable among them. Some patients may progress slowly over the course of many years or decades with minimal impact on the quality of vision, whereas others may have aggressive disease with rapid rates of change that can eventually result in blindness or substantial impairment unless appropriate interventions take place. Therefore, the evaluation of rates of change in glaucoma is essential to allow proper allocation of resources towards patients who are most likely to develop impairment [38]. Even though optic disc stereophotographs have been considered the gold standard for evaluation of structural damage in glaucoma, evaluation of rates of structural change using stereophotographs is difficult because of the qualitative and subjective nature of this assessment [39]. Therefore, imaging technologies capable of quantitatively and objectively measure structural losses have the potential to play an important role for monitoring glaucomatous progression over time and assessing rates of change.

Two different methods have been proposed to identify OCT structural deterioration over time using different approaches to separate true change from test-retest variability: event-based versus trend-based methods. In eventbased methods, the differences in RNFL thicknesses of the current tests are compared with those of previously established baseline examinations, and if they exceed a certain threshold in a specific number of test locations, significant structural progression is declared to have occurred. These thresholds usually are based on test-retest variability levels obtained from patients followed over relatively short periods in which there is reasonable assurance that the disease has not progressed. This approach has recently been introduced into the commercially available guided progression analysis (GPA) software of the Cirrus OCT. In trend-based analyses, RNFL thicknesses of all tests available during the follow-up period of interest are analyzed for the presence of statistically significant change over time, usually with a linear regression approach. Unlike the event-based method, trend analysis relates the magnitude of change over time to the long-term variability observed within the individual data series and does not rely on previously established thresholds. A potential advantage of trend-based methods is that they can provide estimates of rates of structural deterioration over time. These estimates are essential to identify those patients with fast disease progression that could be at higher risk for developing functional impairment as a result of the disease.

A recent cohort study compared the ability of SD-OCT and TD-OCT to detect progressive RNFL thinning by trend analysis and found that SD-OCT was able to detect progression sooner and with greater frequency than TD-OCT [2]. In addition, the agreement with visual field progression was better and the range of variation of rates of change was narrower with SD-OCT. Another recent report demonstrated progressive age-related loss of average RNFL thickness with longitudinal SD-OCT imaging, at a rate of $-0.52 \mu \mathrm{m} /$ year [40]. This finding suggests that detecting a significant negative trend alone may not be sufficient to define glaucomatous progression and that its interpretation should always be made with reference to the normal ranges of age-related decline of RNFL thickness.

The potential use of OCT in detecting glaucomatous progression with an event-based approach has also been evaluated [7]. The study showed that TD-OCT was more sensitive than standard automated perimetry (SAP) for detection of progression. Twenty-two percent of eyes had a significant change measured by TD-OCT without corresponding deterioration of the visual fields. However, it was not clear whether these eyes were true progressors undetected by visual fields or false positives due to variability of 
A

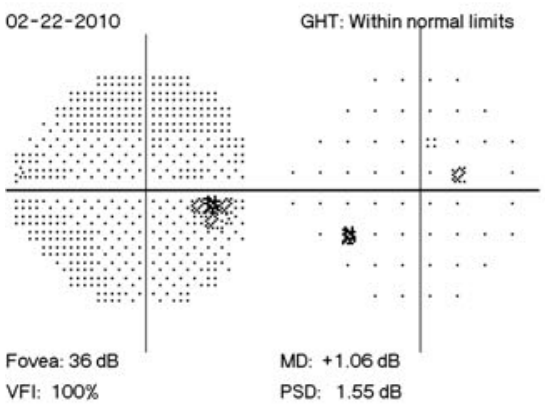

$\mathrm{CSFI}=17 \%$
RNFL thickness

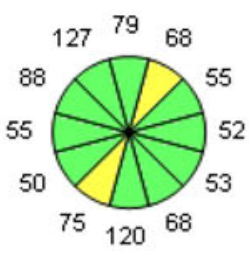

2009

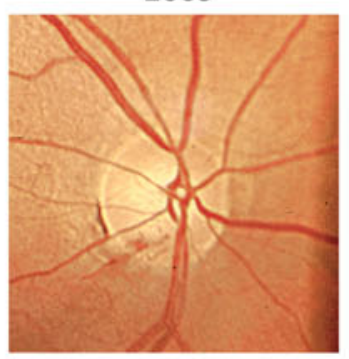

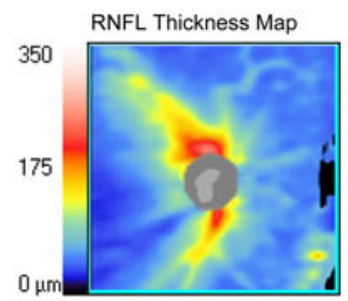

2010

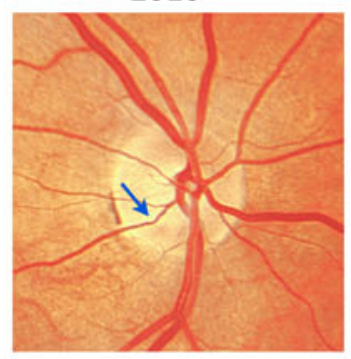

B

Graytone

Pattern Deviation
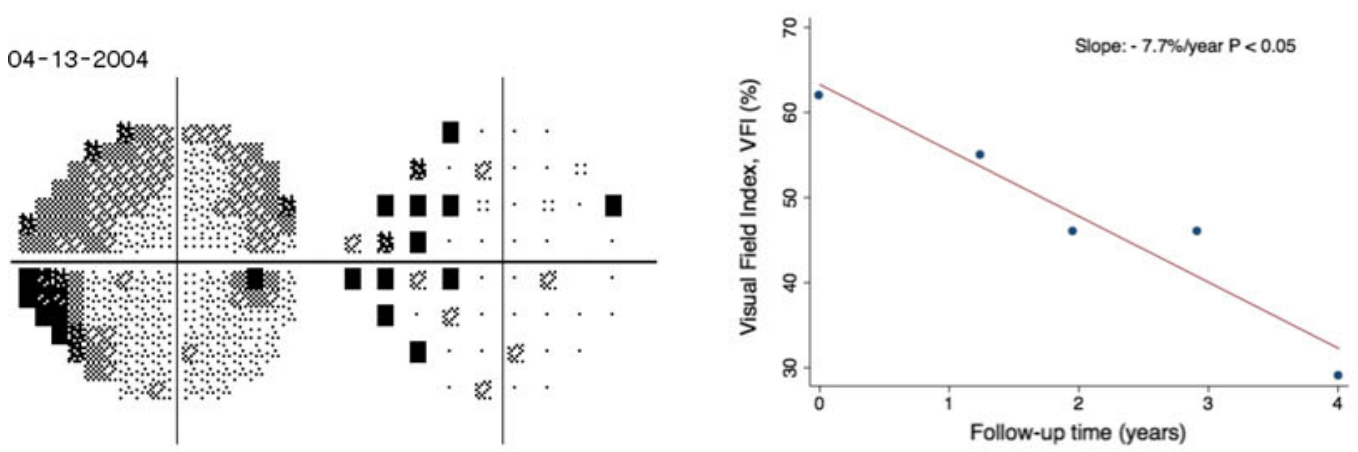

07-12-2005
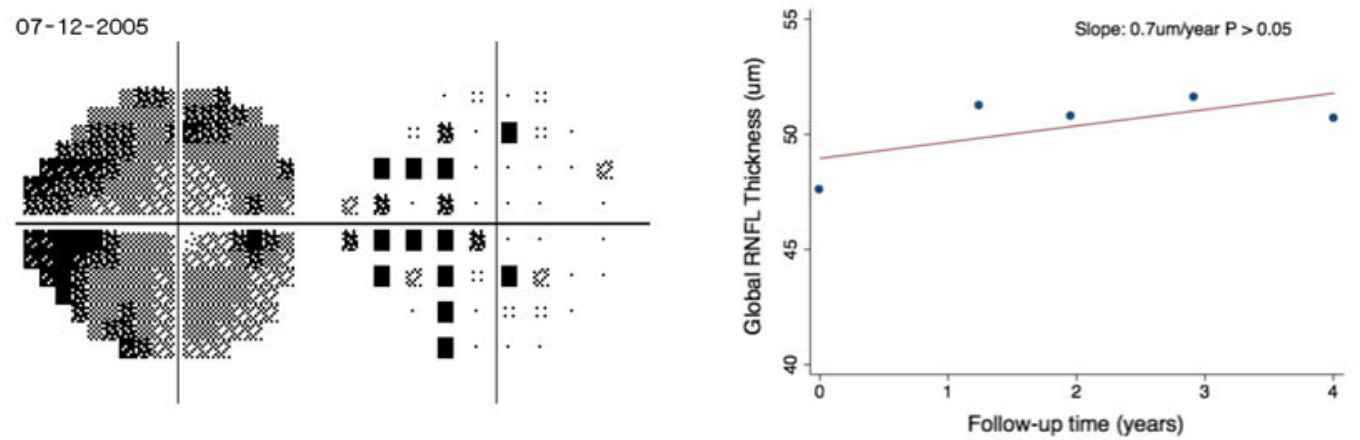

03-14-2007
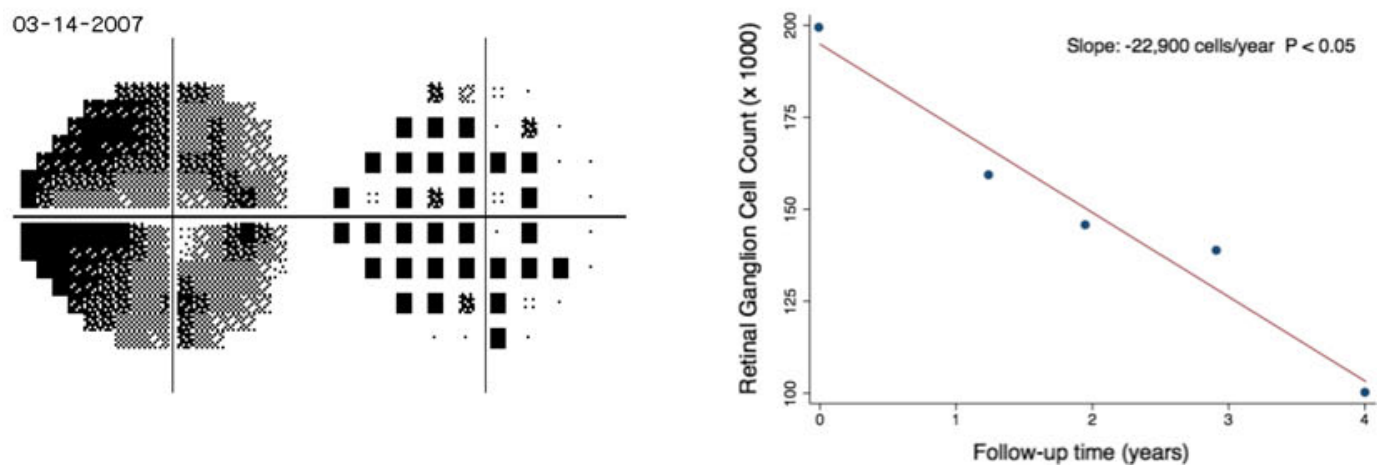
4Fig. 1 Examples of detection of glaucomatous damage and estimation of rates of disease progression using a combined structure and function approach. a Example of an eye with pre-perimetric glaucomatous damage. The eye had evidence of progressive neuroretinal rim thinning over time (arrow) with corresponding RNFL loss on SD-OCT. The visual field exam still had all parameters within statistically normal limits. The CSFI was $17 \%$, indicating a loss of $17 \%$ of RGC compared to the age-expected normal number. b Example of an eye showing severe glaucomatous damage and progressive visual field loss over time. The parameter visual field index showed a deterioration of $-7.7 \% /$ year $(P<0.05)$. Despite the clear visual field progression, longitudinal evaluation with SD-OCT did not show change in RNFL parameters. The use of a combined approach to evaluate the rate of RGC loss, however, estimated a decline of $-22,900$ cells/year, which was statistically significant $(P<0.05) . R N F L$ retinal nerve fiber layer, $C S F I$ combined structure and function index, $V F I$ visual field index, $M D$ mean deviation, $P S D$ pattern standard deviation, GHT glaucoma hemifield test

the measurements or age-related thinning of the RNFL. Recently, a study evaluated the ability of Cirrus OCT to detect RNFL progression using the RNFL thickness change map, a component of the GPA that provides an event-based analysis of RNFL progression based on serial thickness maps [41]. The software automatically aligns the registered baseline and follow-up OCT images so that the same pixel locations can be measured for change. The difference in RNFL thickness of an individual pixel between the baseline and the follow-up maps is then compared to a normative database of test-retest variability of that particular pixel to evaluate the chance of progression. The study found that the most common pattern of RNFL progression was widening of RNFL defects, followed by development of new RNFL defects and then deepening of RNFL defects. The authors suggested that Cirrus' RNFL thickness map analysis could be used as an alternative to red-free RNFL photography for the detection of new defects and to discern the pattern of RNFL progression in glaucoma.

\section{Structure and Function Relationship}

Frequent disagreements are seen when structural and functional tests are used to monitor glaucomatous patients. While SAP has relatively low sensitivity to identify progression at initial stages of the disease, structural assessment often performs poorly to identify change at advanced stages of damage [42]. Differences in performance of structural and functional tests have been recently investigated in a study comparing structural and functional measurements to estimates of RGC counts in glaucoma [42]. In that study, analysis of the relationship between visual field data and RGC counts indicated that, at early stages of the disease, significant losses of RGCs correspond to relatively small changes in visual field parameters. This finding agrees with the large amount of evidence indicating that progressive optic disc or RNFL changes can frequently be seen before the appearance of statistically significant defects on SAP [1•, 3, 6, 7, 43-47].

The relatively poor performance of visual fields in detecting early glaucomatous damage seems to be explained, at least in part, by the measurement scale used for perimetric testing. Scaling of perimetric stimulus intensities has been incorporated into standard perimetric testing, where the stimulus intensities are scaled by a logarithmic transformation to decibel units of attenuation for both the intensity staircase procedure for threshold measurements as well as for the report of the final threshold intensity. Several investigators have suggested that such scaling may introduce an artifactual relationship between structural and functional measurements in glaucoma [44, 46, 48, 49••, 50, 51]. The logarithmic scale accentuates sensitivity changes in the visual field at low decibel values and minimizes changes at high decibel levels, so that perimetry may be more suitable for detection of moderate to severe damage. In contrast, analysis of the relationship between RNFL thickness and estimated RGC counts has indicated that imaging instruments could be used to gauge information on rates of neural loss in early disease, when SAP evaluation can be misleading. At moderate to severe stages of the disease, evaluation of progressive damage with SD-OCT becomes less helpful as the instrument reaches a floor level where it cannot detect further changes.

A recent review by Harwerth et al. [52・•] supports these concepts. In fact, they demonstrated that structural and functional tests are in agreement as long as one uses appropriate measurement scales for neural and sensitivity losses and considers factors such as the effect of aging and eccentricity on estimates of neural losses. In a series of investigations, they demonstrated that estimates of RGC losses obtained from SAP agreed closely with estimates of RGC losses obtained from RNFL assessment by OCT [52・•]. The results of their model provided a common domain for expressing results of structural and functional tests in the same unit, that is, ganglion cell counts.

\section{The Combined Structure and Function Index}

A CSFI was described by Medeiros et al. [49••] with the purpose of merging the results of structural and functional tests into a single index that could be used for diagnosis, staging, and detection of progression in glaucoma. The index uses estimates of RGC counts obtained by previously derived empirical formulas. The estimates of RGC counts are obtained from two sources: one structural (RNFL thickness assessment by OCT), and one functional (visual field testing by SAP). These estimates are then combined using a weighted average to provide a single estimate of 
RGC count for a particular eye. For each eye, the CSFI represents the percent estimate of RGC loss compared with the age-expected number of RGCs. By combining structural and functional tests into a single estimate of RGC loss, the index provides a very intuitive parameter to be used in clinical practice. The CSFI has been shown to perform better than isolated structural and functional parameters for diagnosing and staging glaucomatous damage. Medeiros et al. [49.•] evaluated the CSFI performance in a cross-sectional study involving 333 glaucomatous eyes and 165 healthy subjects. From the 333 glaucomatous eyes, 295 (89\%) had perimetric glaucoma and $38(11 \%)$ had pre-perimetric glaucoma. The mean CSFIs, representing the mean estimated percent loss of RGCs, were 41 and $17 \%$ in the perimetric and pre-perimetric groups, respectively. The index had excellent diagnostic performance to detect glaucomatous eyes, with an area under the ROC curve (AUC) of 0.94 . Moreover, the index was also able to successfully detect eyes with pre-perimetric glaucoma, with AUC of 0.85 . This compared favorably to the usual parameters provided by SAP and SD-OCT. The CSFI was also able to successfully stage different degrees of glaucomatous damage, which is an essential requirement for any method proposed to detect disease progression over time. To separate eyes with early from moderate visual field loss, the CSFI had AUC of 0.94 compared to only 0.77 for SD-OCT average thickness $(P<0.001)$. Similarly, for separating moderate from advanced glaucomatous field loss, the AUC of the CSFI was 0.96, which was again significantly better than that for average RNFL thickness (AUC $=0.70 ; P<0.001$ ).

Approaches combining structure and function can take advantage of the different performance of these tests according to the stage of damage in order to provide a reliable method for detecting change throughout the spectrum of the disease. It is important to emphasize that an optimal method for detecting glaucomatous progression should not only give an indication of whether or not the eye is changing over timebut should also estimate the rate of deterioration. Estimates of RGC counts from a combination of structural and functional tests have been shown to be able to detect glaucomatous progression and estimate rates of disease deterioration [53.•]. In a longitudinal study of 213 eyes followed for an average of 4.5 years, 47 (22.1\%) showed statistically significant rates of estimated RGC loss that were faster than the age-expected decline. The mean rate of estimated RGC loss in these eyes was $-33,369$ cells/year (range: $-8,332$ cells/year to $-80,636$ cells/year). In addition, estimates of RGC losses detected a significantly larger number of progressing eyes compared to isolated measures of function and structure at the same specificity level [53・•]. Figure 1 illustrates the results of structural and functional evaluation in two eyes with different stages of glaucomatous damage. Case A had a RNFL defect detected by the OCT in the presence of a normal visual field. The CSFI for this patient was able to successfully detect the presence of damage and showed an estimated amount of RGC loss of $17 \%$. Case B presented severe and progressive glaucomatous damage as shown by the visual fields; however, the RNFL average thickness measured by OCT was not able to detect the disease progression. Estimates of RGC counts showed clear progression in this case. These findings illustrate the importance of the single index combining both structural and functional evaluations for detecting and monitoring glaucomatous damage throughout the spectrum of the disease.

Other approaches have been suggested to combine structural and functional tests to detect glaucomatous progression, including the use of Bayesian methodologies to allow combination of different tests [54, 55]. These approaches are effective in combining results of different tests to improve the estimates of rate of change and have the advantage of being capable of incorporating other covariates, such as demographic and clinical risk factors, to increase the accuracy and precision of the estimates [55]. However, Bayesian analyses have the disadvantage of not being intuitive for the majority of clinicians.

\section{Conclusion}

Recent advances in OCT have resulted in major improvements in the ability of this technology to assess structural damage in glaucoma. The differences in performance of structural and functional tests according to the stage of disease indicate the strong need for combined approaches for the evaluation of progressive damage in glaucoma. A recently described approach that estimates rates of RGC loss from a combination of structural and functional tests offers a promising method for disease diagnosis and staging, detecting progression and estimating rates of glaucomatous deterioration.

Acknowledgments This study was supported in part by National Institutes of Health/National Eye Institute Grants EY021818 (F.A.M.) and Brazilian National Research Council-CNPq Grant 200178/2012-1 (D.M.F).

Disclosure D. Meira-Freitas: none; R. Lisboa: none; F.A. Medeiros: financial support from Carl-Zeiss Meditec, and Heidelberg Engineering.

\section{References}

Papers of particular interest, published recently, have been highlighted as:

- Of importance,

•- Of major importance 
1. • Leung CK, Cheung CY, Weinreb RN, et al. Evaluation of retinal nerve fiber layer progression in glaucoma: a study on optical coherence tomography guided progression analysis. Investig Ophthalmol Vis Sci. 2010;51(1):217-22. This study shows the potential benefit of the newer spectral-domain optical coherence technology compared to time-domain technology for detection of glaucomatous progression.

2. Leung CK, Cheung CY, Weinreb RN, et al. Evaluation of retinal nerve fiber layer progression in glaucoma: a comparison between the fast and the regular retinal nerve fiber layer scans. Ophthalmology. 2011;118(4):763-7.

3. Medeiros FA, Alencar LM, Zangwill LM, et al. Prediction of functional loss in glaucoma from progressive optic disc damage. Arch Ophthalmol. 2009;127(10):1250-6.

4. Medeiros FA, Weinreb RN, Sample PA, et al. Validation of a predictive model to estimate the risk of conversion from ocular hypertension to glaucoma. Arch Ophthalmol. 2005;123(10): 1351-60.

5. Medeiros FA, Zangwill LM, Alencar LM, et al. Detection of glaucoma progression with stratus OCT retinal nerve fiber layer, optic nerve head, and macular thickness measurements. Invest Ophthalmol Vis Sci. 2009;50(12):5741-8.

6. Strouthidis NG, Scott A, Peter NM, Garway-Heath DF. Optic disc and visual field progression in ocular hypertensive subjects: detection rates, specificity, and agreement. Invest Ophthalmol Vis Sci. 2006;47(7):2904-10.

7. Wollstein G, Schuman JS, Price LL, et al. Optical coherence tomography longitudinal evaluation of retinal nerve fiber layer thickness in glaucoma. Arch Ophthalmol. 2005;123(4):464-70.

8. Nassif N, Cense B, Park B, et al. In vivo high-resolution videorate spectral-domain optical coherence tomography of the human retina and optic nerve. Opt Express. 2004;12(3):367-76.

9. Wojtkowski M, Srinivasan V, Fujimoto JG, et al. Three-dimensional retinal imaging with high-speed ultrahigh-resolution optical coherence tomography. Ophthalmology. 2005;112(10):1734-46.

10. Leung CK, Cheung CY, Weinreb RN, et al. Retinal nerve fiber layer imaging with spectral-domain optical coherence tomography: a variability and diagnostic performance study. Ophthalmology. 2009;116(7):1257-63.

11. Schuman JS. Spectral domain optical coherence tomography for glaucoma (an AOS thesis). Trans Am Ophthalmol Soc. 2008;106: 426-58.

12. DeBuc DC, Somfai GM, Ranganathan S, et al. Reliability and reproducibility of macular segmentation using a custom-built optical coherence tomography retinal image analysis software. J Biomed Opt. 2009;14(6):064023.

13. Fabritius T, Makita S, Miura M, et al. Automated segmentation of the macula by optical coherence tomography. Opt Express. 2009; 17(18):15659-69.

14. Garvin MK, Abramoff MD, Wu X, et al. Automated 3-D intraretinal layer segmentation of macular spectral-domain optical coherence tomography images. IEEE Trans Med Imaging. 2009;28(9):1436-47.

15. Mwanza JC, Oakley JD, Budenz DL, et al. Macular ganglion cellinner plexiform layer: automated detection and thickness reproducibility with spectral domain-optical coherence tomography in glaucoma. Invest Ophthalmol Vis Sci. 2011;52(11):8323-9.

16. Tan O, Chopra V, Lu AT, et al. Detection of macular ganglion cell loss in glaucoma by Fourier-domain optical coherence tomography. Ophthalmology. 2009;116(12):2305-14.

17. Wang M, Hood DC, Cho JS, et al. Measurement of local retinal ganglion cell layer thickness in patients with glaucoma using frequency-domain optical coherence tomography. Arch Ophthalmol. 2009;127(7):875-81.

18. Drexler W, Fujimoto JG. State-of-the-art retinal optical coherence tomography. Prog Retin Eye Res. 2008;27(1):45-88.
19. Blumenthal EZ, Parikh RS, Pe'er J, et al. Retinal nerve fibre layer imaging compared with histological measurements in a human eye. Eye (Lond). 2009;23(1):171-5.

20. Chen TC, Cense B, Miller JW, et al. Histologic correlation of in vivo optical coherence tomography images of the human retina. Am J Ophthalmol. 2006;141(6):1165-8.

21. Strouthidis NG, Grimm J, Williams GA, et al. A comparison of optic nerve head morphology viewed by spectral domain optical coherence tomography and by serial histology. Invest Ophthalmol Vis Sci. 2010;51(3):1464-74.

22. Leite MT, Rao HL, Weinreb RN, et al. Agreement among spectral-domain optical coherence tomography instruments for assessing retinal nerve fiber layer thickness. Am J Ophthalmol. 2011;151(1):85-92.

23. Rao HL, Zangwill LM, Weinreb RN, et al. Comparison of different spectral domain optical coherence tomography scanning areas for glaucoma diagnosis. Ophthalmology. 2010;117(9):1692-9.

24. Gonzalez-Garcia AO, Vizzeri G, Bowd C, et al. Reproducibility of RTVue retinal nerve fiber layer thickness and optic disc measurements and agreement with Stratus optical coherence tomography measurements. Am J Ophthalmol. 2009;147(6):1067-74.

25. Sung KR, Kim JS, Wollstein G, et al. Imaging of the retinal nerve fibre layer with spectral domain optical coherence tomography for glaucoma diagnosis. Br J Ophthalmol. 2011;95(7):909-14.

26. Leite MT, Rao HL, Zangwill LM, et al. Comparison of the diagnostic accuracies of the Spectralis, Cirrus, and RTVue optical coherence tomography devices in glaucoma. Ophthalmology. 2011;118(7):1334-9.

27. Hwang YH, Kim YY. Glaucoma diagnostic ability of quadrant and clock-hour neuroretinal rim assessment using cirrus HD optical coherence tomography. Invest Ophthalmol Vis Sci. 2012; 53(4):2226-34.

28. Leung CK, Ye C, Weinreb RN, et al. Retinal nerve fiber layer imaging with spectral-domain optical coherence tomography a study on diagnostic agreement with Heidelberg Retinal Tomograph. Ophthalmology. 2010;117(2):267-74.

29. Li S, Wang X, Li S, et al. Evaluation of optic nerve head and retinal nerve fiber layer in early and advance glaucoma using frequency-domain optical coherence tomography. Graefe's Arch Clin Exp Ophthalmol. 2010;248(3):429-34.

30. Mwanza JC, Oakley JD, Budenz DL, Anderson DR. Ability of cirrus HD-OCT optic nerve head parameters to discriminate normal from glaucomatous eyes. Ophthalmology. 2011;118(2):241-8.

31. Sehi M, Grewal DS, Sheets CW, Greenfield DS. Diagnostic ability of Fourier-domain vs time-domain optical coherence tomography for glaucoma detection. Am J Ophthalmol. 2009;148(4):597-605.

32. Kotowski J, Folio LS, Wollstein G, et al. Glaucoma discrimination of segmented cirrus spectral domain optical coherence tomography (SD-OCT) macular scans. Br J Ophthalmol. 2012; 96(11):1420-5.

33. Na JH, Sung KR, Baek S, et al. Macular and retinal nerve fiber layer thickness: which is more helpful in the diagnosis of glaucoma? Invest Ophthalmol Vis Sci. 2011;52(11):8094-101.

34. Lisboa R, Leite MT, Zangwill LM, et al. Diagnosing preperimetric glaucoma with spectral domain optical coherence tomography. Ophthalmology. 2012;119(11):2261-9.

35. Wang X, Li S, Fu J, et al. Comparative study of retinal nerve fibre layer measurement by RTVue OCT and GDx VCC. Br J Ophthalmol. 2011;95(4):509-13.

36. Vizzeri G, Weinreb RN, Gonzalez-Garcia AO, et al. Agreement between spectral-domain and time-domain OCT for measuring RNFL thickness. Br J Ophthalmol. 2009;93(6):775-81.

37. Kim JS, Ishikawa H, Sung KR, et al. Retinal nerve fibre layer thickness measurement reproducibility improved with spectral domain optical coherence tomography. Br J Ophthalmol. 2009; 93(8):1057-63. 
38. Mansouri K, Leite MT, Medeiros FA, et al. Assessment of rates of structural change in glaucoma using imaging technologies. Eye (Lond). 2011;25(3):269-77.

39. Jampel HD, Friedman D, Quigley H, et al. Agreement among glaucoma specialists in assessing progressive disc changes from photographs in open-angle glaucoma patients. Am J Ophthalmol. 2009;147(1):39-44.

40. Leung $\mathrm{CK}, \mathrm{Yu} \mathrm{M}$, Weinreb RN, et al. Retinal nerve fiber layer imaging with spectral-domain optical coherence tomography: a prospective analysis of age-related loss. Ophthalmology. 2012; 119(4):731-7.

41. Leung CK, Yu M, Weinreb RN, et al. Retinal nerve fiber layer imaging with spectral-domain optical coherence tomography: patterns of retinal nerve fiber layer progression. Ophthalmology. 2012;119(9):1858-66.

42. Medeiros FA, Zangwill LM, Bowd C, et al. The structure and function relationship in glaucoma: implications for detection of progression and measurement of rates of change. Invest Ophthalmol Vis Sci. 2012;53(11):6939-46.

43. Harwerth RS, Carter-Dawson L, Smith EL 3rd, et al. Neural losses correlated with visual losses in clinical perimetry. Invest Ophthalmol Vis Sci. 2004;45(9):3152-60.

44. Hood DC, Kardon RH. A framework for comparing structural and functional measures of glaucomatous damage. Prog Retin Eye Res. 2007;26(6):688-710.

45. Kass MA, Heuer DK, Higginbotham EJ, et al. The Ocular Hypertension Treatment Study: a randomized trial determines that topical ocular hypotensive medication delays or prevents the onset of primary open-angle glaucoma. Arch Ophthalmol. 2002; 120(6):701-13.

46. Medeiros FA, Alencar LM, Zangwill LM, et al. The relationship between intraocular pressure and progressive retinal nerve fiber layer loss in glaucoma. Ophthalmology. 2009;116(6):1125-33.

47. Miglior S, Zeyen T, Pfeiffer N, et al. Results of the European Glaucoma Prevention Study. Ophthalmology. 2005;112(3):366-75.

48. Harrell FEJ. Regression modelling strategies with applications to linear models, logistic regression and survival analysis. New York: Springer; 2001.
49. • Medeiros FA, Lisboa R, Weinreb RN, et al. A combined index of structure and function for staging glaucomatous damage. Arch Ophthalmol. 2012;130(9):1107-16. This is the first study in the literature describing a single index for estimating retinal ganglion cell counts from a combination of structural and functional tests in glaucoma. The index was shown to successfully diagnose and stage the disease.

50. Garway-Heath DF, Caprioli J, Fitzke FW, Hitchings RA. Scaling the hill of vision: the physiological relationship between light sensitivity and ganglion cell numbers. Invest Ophthalmol Vis Sci. 2000;41(7):1774-82.

51. Swanson WH, Felius J, Pan F. Perimetric defects and ganglion cell damage: interpreting linear relations using a two-stage neural model. Invest Ophthalmol Vis Sci. 2004;45(2):466-72.

52. •• Harwerth RS, Wheat JL, Fredette MJ, Anderson DR. Linking structure and function in glaucoma. Prog Retin Eye Res. 2010;29(4):249-71. Excellent review of the relationship between results of different structural and functional tests in glaucoma supported by extensive experimental studies.

53. • Medeiros FA, Zangwill LM, Anderson DR, et al. Estimating the rate of retinal ganglion cell loss in glaucoma. Am J Ophthalmol. 2012;154(5):814-24. First study to develop and apply a technique to estimate retinal ganglion cell counts for detecting glaucomatous progression and estimating rates of disease deterioration. The estimated retinal ganglion cell counts were obtained from a combination of structural and functional tests and performed better than conventional parameters for detection of change over time.

54. Medeiros FA, Leite MT, Zangwill LM, Weinreb RN. Combining structural and functional measurements to improve detection of glaucoma progression using Bayesian hierarchical models. Invest Ophthalmol Vis Sci. 2011;52(8):5794-803.

55. Russell RA, Malik R, Chauhan BC, et al. Improved estimates of visual field progression using bayesian linear regression to integrate structural information in patients with ocular hypertension. Invest Ophthalmol Vis Sci. 2012;53(6):2760-9. 